\title{
A DUSTY COMPONENT TO THE GASEOUS DEBRIS DISK AROUND THE WHITE DWARF SDSS J1228+1040
}

\author{
C. S. Brinkworth ${ }^{1}$, B. T. GÄnsiCKe ${ }^{2}$, T. R. Marsh $^{2}$, D. W. Hoard ${ }^{1}$, And C. TAPPerT ${ }^{3}$ \\ ${ }^{1}$ Spitzer Science Center, California Institute of Technology, Pasadena, CA 91125, USA \\ ${ }^{2}$ Department of Physics and Astronomy, University of Warwick, Warwick CV4 7AL, UK \\ ${ }^{3}$ Dpto de Astronomía y Astrofísica, Pontificia Universidad Católica de Chile, Casilla 306, Santiago 22, Chile \\ Received 2008 July 23; accepted 2009 February 18; published 2009 April 24
}

\begin{abstract}
We present Infrared Spectrometer And Array Camera (ISAAC) spectroscopy and ISAAC, UKIDSS, and Spitzer Space Telescope broadband photometry of SDSS J1228+1040-a white dwarf for which evidence of a gaseous metal-rich circumstellar disk has previously been found from optical emission lines. The data show a clear excess in the near- and mid-infrared (IR), providing compelling evidence for the presence of dust in addition to the previously identified gaseous debris disk around the star. The IR excess can be modeled in terms of an optically thick but geometrically thin disk. We find that the inner disk temperatures must be relatively high $(\sim 1700 \mathrm{~K})$ in order to fit the spectral energy distribution in the near-IR. These data provide the first evidence for the coexistence of both gas and dust in a disk around a white dwarf, and show that their presence is possible even around moderately hot $(\sim 22,000 \mathrm{~K})$ stars.
\end{abstract}

Key words: circumstellar matter - infrared: stars - stars: individual (SDSS J1228+1040) - white dwarfs

\section{INTRODUCTION}

Despite the numerous extrasolar planets discovered over the last decade, the fate of these systems during the final stages of the parent star's evolution is still uncertain. A clue to their destiny was discovered during a search for cool companions to white dwarfs. G29-38 was found to display a strong nearinfrared (IR) excess (Zuckerman \& Becklin 1987), but deep imaging and asteroseismological studies of the system ruled out the presence of a brown dwarf companion. Mid-IR photometry showed that this excess is even more pronounced at $10 \mu \mathrm{m}$ suggesting that the emission is due to dust around the white dwarf (Graham et al. 1990; Telesco et al. 1990; Tokunaga et al. 1990). Spitzer Space Telescope observations spectroscopically confirmed the presence of circumstellar dust around G29-38 (Reach et al. 2005), which has since been modeled as a flat, opaque, circumstellar disk (von Hippel et al. 2007). The atmosphere of G29-38 has been found to be enriched with metals, implying that the star is currently accreting (Koester et al. 1997), probably from this dusty disk.

The more likely origin for the disk is the tidal disruption of either comets or asteroids (Debes \& Sigurdsson 2002; Jura 2003), with asteroids the more probable candidates, as they can explain the large amount of metals accreted by the white dwarfs. Comets are also dynamically less favorable, given their eccentric orbits and long orbital periods. The approach used by von Hippel et al. (2007) to model the disk was based on a simple, optically thick, geometrically thin dusty disk model described in Jura (2003).

A further 11 white dwarfs have been found to display a similar IR excess (Becklin et al. 2005; Kilic et al. 2005, 2006; von Hippel et al. 2007; Jura et al. 2007a, 2009; Farihi et al. 2008, 2009), most of which are cool, metal-rich DAZ white dwarfs, with effective temperatures $T_{\text {eff }}<15,000 \mathrm{~K}$ (with the exceptions of PG 1015+161, which has $T_{\text {eff }} \sim 19,300 \mathrm{~K}$, and PG 1457-086 with $T_{\text {eff }} \sim 20,400 \mathrm{~K}$ ), leading Kilic et al. (2006) to suggest that dusty disks around white dwarfs should be sublimated for $T_{\text {eff }} \geqslant 16,000-20,000 \mathrm{~K}$. More recently, Gänsicke et al. (2006, 2007, 2008) identified three more moderately hot white dwarfs showing double-peaked calcium lines indicative of a rotating circumstellar debris disk: SDSS J122859.93+104032.9 (hereafter SDSS 1228+1040), SDSS J104341.53+085558.2, and SDSS J084539.17+225728.0.

Here we report the detection of a significant IR excess in one of these systems, SDSS 1228+1040. The white dwarf was identified by Gänsicke et al. (2006) in Data Release 6 of the Sloan Digital Sky Survey (Adelman-McCarthy et al. 2006). Gänsicke et al. (2006) noted very unusual emission lines of the Ca II 850-866 nm triplet, as well as weaker emission lines of Fe II at $502 \mathrm{~nm}$ and $517 \mathrm{~nm}$. The line profiles of the Ca II triplet display a double-peaked morphology, indicative of a gaseous, rotating disk structure. Time-resolved spectroscopy ruled out any significant radial velocity variations, and the absence of Balmer and helium emission lines implied that the gaseous disk around the white dwarf must be extremely deficient in volatile materials, ruling out the presence of a stellar binary companion. The velocity of the $\mathrm{Ca}$ II line peaks indicate that the outer radius of the gaseous circumstellar disk is $\simeq 1.2 R_{\odot}$.

\section{OBSERVATIONS AND DATA REDUCTION}

\subsection{Spitzer Space Telescope Data}

We were awarded $2.4 \mathrm{hr}$ of Spitzer Space Telescope time in Cycle 4 (P40048), plus $3.0 \mathrm{hr}$ in Cycle 5 (P50118) to search for a dusty component to the gaseous circumstellar disk around SDSS 1228+1040 (see Table 1 for a full list of observations). We obtained Infrared Array Camera (IRAC; Fazio et al. 2004) data in all four channels $(3.6-8.0 \mu \mathrm{m})$, Infrared Spectrograph (IRS; Houck et al. 2004) Blue Peak-Up imaging at $16 \mu \mathrm{m}$, and Multiband Imaging Photometer (MIPS; Rieke et al. 2004) imaging at $24 \mu \mathrm{m}$.

The IRAC data reduction was carried out on the Basic Calibrated Data (BCD) frames as follows. We corrected all of the $\mathrm{BCDs}$ for array-location dependence using the corresponding correction frames provided by the Spitzer Science Center (SSC). These were applied after uncorrecting the array-locationdependence frames for pixel distortion in order to prevent a repeat correction for pixel distortion when creating the mosaic. The array-location-corrected BCDs were then all combined using the standard SSC software, MOsaicker and Point source EXtraction (MOPEX; Makovoz et al. 2006) with dual-outlier rejection to create a single mosaicked image for each channel. 


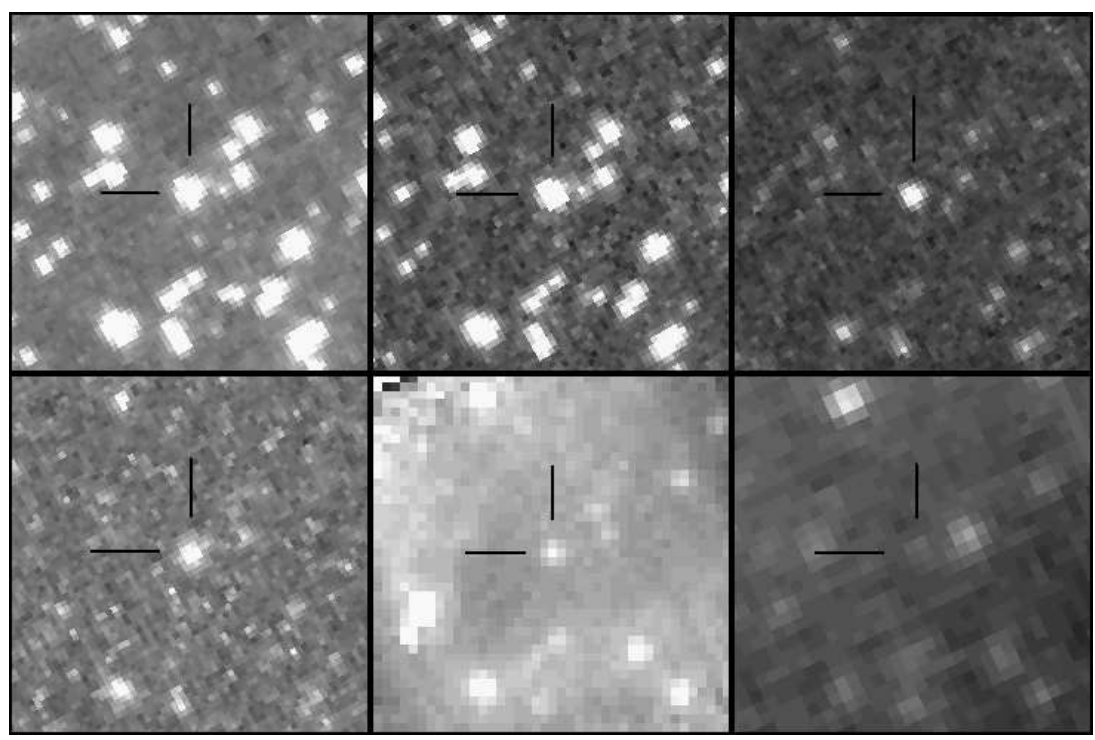

Figure 1. Mosaicked images for SDSS 1228+1040: north, up; east, left. Top row, left to right: IRAC-1 (3.6 $\mu \mathrm{m})$, IRAC-2 (4.5 $\mu \mathrm{m})$, and IRAC-3 (5.8 $\mu \mathrm{m})$. Bottom row, left to right: IRAC-4 $(8.0 \mu \mathrm{m})$, IRS PU $(16 \mu \mathrm{m})$, and MIPS-24 $(24 \mu \mathrm{m})$. Pixel scales are $1^{\prime \prime} 22$ pixel $^{-1}, 1^{\prime \prime} 8$ pixel $^{-1}$, and 2 ." 45 pixel $^{-1}$ for the IRAC, IRS PU, and MIPS-24 mosaics, respectively. The telescope pointing is good to $<0$ '.5

Table 1

Observation Log for Spitzer Space Telescope Data

\begin{tabular}{lccccc}
\hline \hline $\begin{array}{l}\text { Instrument/ } \\
\text { Channel }\end{array}$ & $\begin{array}{c}\text { Wavelength } \\
(\mu \mathrm{m})\end{array}$ & AOR Key & $\begin{array}{c}\text { Total Integration Time } \\
(\mathrm{s})\end{array}$ & Date & Pipeline \\
\hline IRAC Ch. 1 & 5.550 & 22247936 & 1000 & 2007 Jun 30 & S16.1 \\
IRAC Ch. 2 & 4.493 & 22247936 & 1000 & 2007 Jun 30 & S16.1 \\
IRAC Ch. 3 & 5.731 & 22247936 & 1000 & 2007 Jun 30 & S16.1 \\
IRAC Ch. 4 & 7.872 & 22247936 & 1000 & 2007 Jun 30 & S16.1 \\
IRS Blue Peak-Up & 15.6 & 22248192 & 3450 & 2007 Jun 16 & S16.1 \\
MIPS-24 & 23.68 & 25459200 & 3012 & 2008 Jul 25 & S18.1 \\
MIPS-24 & 23.68 & 25459456 & 3012 & 2008 Jul 25 & S18.1 \\
MIPS-24 & 23.68 & 25459712 & 3012 & 2008 Jul 25 & S18.1 \\
\hline
\end{tabular}

The mosaic pixel scales were set to the default $1^{\prime \prime} .22$ pixel $^{-1}$. As a check on our reduction method, we also split the BCDs into batches of seven frames, creating a mosaic from each batch in order to enable us to test the repeatability of the photometry and calculate an independent estimate of our uncertainties.

The IRS Peak-Up imaging frames were combined in much the same way, using MOPEX to combine the BCDs with dualoutlier rejection. Again, we created a mosaic from both the entire data set, and from subsets of the data, so that we could test the repeatability of the photometry and derive realistic photometric uncertainties from our data. The pixel scales for the mosaics were set to the default 1".8 $\mathrm{pixel}^{-1}$. A similar method was applied to the MIPS data, where we created a separate mosaic for each of the Exposure ID sets (35 BCDs in each set). This gave us 21 mosaics in total, all of which were created using multiframe temporal outlier rejection and the default pixel scale of 2".45 pixel $^{-1}$. The mosaicked images for all of the Spitzer data, with SDSS 1228+1040 marked, can be found in Figure 1.

We carried out aperture photometry on our IRAC and IRS PU mosaics using IRAF, using apertures of 3.0 pixels for the IRAC mosaics and 2.0 pixels for the IRS mosaics. The sky subtraction was applied using an annulus of 10-20 pixels in radius. In IRAC channels 1 and 2 there were background sources included in the sky annuli. IRAF rejects bright pixels in the sky annuli, but we carried out a double-check of the sky levels using nearby apertures, and found them to be virtually identical to the levels originally measured. The photometry was then converted from $\mathrm{MJy} \mathrm{sr}^{-1}$ to $\mu \mathrm{Jy}$ and aperture-corrected to the calibration aperture sizes using the standard aperture corrections provided by the SSC. The IRAC channel 1 photometry was further corrected for pixel-phase dependence in accordance with SSC recommendations. No color correction was applied since we quote the isophotal effective wavelengths, thus eliminating most of the color dependency of the flux calibration and rendering the magnitude of the correction negligible compared to our uncertainties.

We derived our uncertainty estimates for the IRAC and IRS data using the standard deviation of the scatter in the subset mosaics at each wavelength. These were then added in quadrature to the absolute calibration estimates of the IRAC and IRS instruments (Reach et al. 2005, SSC Web site ${ }^{4}$ ).

Looking at the MIPS mosaics, we found that the target is very faint, and there is bright source within $\sim 5-6$ pixels. While it is tempting to suspect that the bright source may, in fact, be the target, the pointing of Spitzer is good to within 0.'5, while the pixel scale of the mosaic is 2 ". 45 pixel $^{-1}$, and so there is no doubt that the fainter of the two sources is SDSS 1228+1040. This is corroborated by matching the positions with respect to the surrounding stars. We used MOPEX to carry out a source extraction and subtraction on each mosaic, removing all of the high signalto-noise ratio $(\mathrm{S} / \mathrm{N})$ sources from the field (including the close, bright neighbor), before carrying out IRAF aperture photometry

\footnotetext{
4 http://ssc.spitzer.caltech.edu/irac/documents/reach2005.pdf
} 
on our lower $\mathrm{S} / \mathrm{N}$, unsubtracted target. We used an aperture of $3^{\prime \prime}$ (1.224 pixels) to extract the photometry, with a background annulus of $20^{\prime \prime}-32^{\prime \prime}$, and applied the standard aperture corrections as given in the MIPS Data Handbook. We double-checked the flux density we obtained by performing MOPEX PRF fitting on our target in the original mosaics. The two methods agreed to within $10 \%$, while the standard deviation in the spread in flux densities from the 21 mosaics gave us uncertainties of $\sim 50 \%$. The final flux density was taken as the unweighted median in the 21 measurements from the aperture photometry.

\subsection{ISAAC Data}

The IR spectroscopic data were taken with the Infrared Spectrometer And Array Camera (ISAAC) mounted at Antu (UT1), VLT, Paranal, Chile. The short-wavelength grism was used in its low-resolution mode at central wavelengths $1.06 \mu \mathrm{m}$ $(z), 1.25 \mu \mathrm{m}(J), 1.65 \mu \mathrm{m}(H)$, and $2.20 \mu \mathrm{m}(K)$. An 0.8 slit width resulted in resolving powers in the range 650-750. The observations were conducted in service mode on two nights, 2007 April $3(J$ and $H$ ) and 2007 April $7(z$ and $K$ ). Flat fields and wavelength calibration with a Xe-Ar lamp (Ar only for the $z$ spectra) were taken at the start of the night, and telluric standards were observed within $1 \mathrm{hr}$ and an air mass difference $\Delta M(z)=0.2$ of the target spectra. The data were obtained as sets of 22 spectra per central wavelength with individual exposure times of $100 \mathrm{~s}$. The individual spectra were taken at different positions on the detector ( $\mathrm{AB}$ cycles plus jittering) to minimize the effect of bad pixels and cosmic rays.

For the reduction, IRAF packages were applied. As first steps, the data were flat-fielded and "straightened" via a twodimensional wavelength calibration, thus correcting for the positional dependence of the dispersion. Corresponding $\mathrm{AB}$ pairs were subtracted from each other, and the resulting data then were combined to a single image. Subsequently, the target and standard spectra were extracted. In the case of the $z$ spectrum it proved necessary to adjust the wavelength calibration using the night sky lines (Rousselot et al. 2000).

The following stars were used as telluric standards: HD $106807\left(\mathrm{~A} 1 \mathrm{~V}, T_{\text {eff }}=9400 \mathrm{~K}\right)$ for the $z$ data, Hip 43699 (B4V, $17,000 \mathrm{~K}$ ) for $J$ and $H$, and Hip 88857 (B3V, 19,000 K) for $K$. The intrinsic lines of the standards were fitted with a Voigt profile and subtracted from the IR spectrum, to yield a pure atmospheric absorption spectrum. For the $z$ data, the intrinsic lines were submerged in atmospheric absorption bands and could not be corrected for. The target spectra were then divided by their corresponding telluric spectra, after the latter had been shifted and scaled to match the position and depths of the atmospheric features in the target data. Finally, the spectra were multiplied with a blackbody corresponding to the effective temperature of the telluric standard, thus recovering the intrinsic spectral energy distribution (SED).

We used the ISAAC $J H K$ acquisition images to perform differential photometry against nearby bright stars that have reliable magnitudes in Two Micron All Sky Survey (2MASS), where we assumed a conservative $10 \%$ error for the IR fluxes derived from these measurements. The JHK magnitudes of SDSS $1228+1040$ obtained in this way were then used to correct the flux level of the ISAAC spectra for slit losses.

\subsection{UKIDSS Data}

SDSS $1228+1040$ is within the footprint of the Large Area Survey within the third Data Release (DR3) of the UKIRT

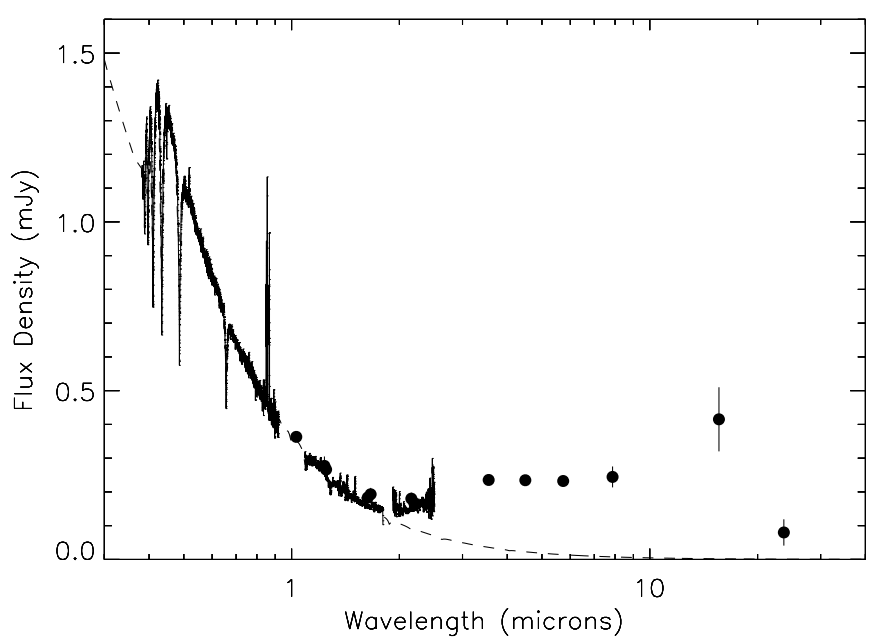

Figure 2. SED for SDSS $1228+1040$ from the ultraviolet to the mid-IR. UV spectra and the white dwarf model (dashed line) are taken from Gänsicke et al. (2006). The near-IR spectroscopy and YJHK photometry were taken with ISAAC/VLT and UKIDSS. The mid-IR observations from 3.6 to $24 \mu \mathrm{m}$ were taken with the Spitzer Space Telescope IRAC, IRS Blue Peak-Up, and MIPS instruments. The SED shows a significant flux density excess from the $K$ band to longer wavelengths, compared to the white dwarf alone.

Table 2

Photometry for SDSS $1228+1040$ from Spitzer and ISAAC

\begin{tabular}{lccc}
\hline \hline $\begin{array}{l}\text { Instrument/ } \\
\text { Band }\end{array}$ & $\begin{array}{c}\text { Wavelength } \\
(\mu \mathrm{m})\end{array}$ & $\begin{array}{c}\text { Flux Density } \\
(\mathrm{mJy})\end{array}$ & $\begin{array}{c}\text { Uncertainty } \\
(\%)\end{array}$ \\
\hline ISAAC $/ J$ & 1.235 & 0.277 & 10 \\
ISAAC $/ H$ & 1.662 & 0.193 & 10 \\
ISAAC $/ K$ & 2.159 & 0.180 & 10 \\
UKIDSS $/ y$ & 1.031 & 0.363 & 1.1 \\
UKIDSS $/ J$ & 1.248 & 0.266 & 1.5 \\
UKIDSS $/ H$ & 1.631 & 0.183 & 2.2 \\
UKIDSS $/ K$ & 2.101 & 0.167 & 3.6 \\
IRAC $/ 1$ & 3.550 & 0.235 & 4.5 \\
IRAC $/ 2$ & 4.493 & 0.235 & 4.1 \\
IRAC $/ 3$ & 5.731 & 0.232 & 7.5 \\
IRAC $/ 4$ & 7.872 & 0.244 & 12.8 \\
IRS PU $/ 16$ & 15.6 & 0.415 & 23.0 \\
MIPS $/ 24$ & 23.68 & 0.080 & 49 \\
\hline
\end{tabular}

Infrared Deep Sky Survey (UKIDSS; Lawrence et al. 2007). We obtained $Y J H K$ aperture magnitudes from the WFCAM archive (Hambly et al. 2008), and converted them to fluxes at the effective wavelengths using the zero-point definitions in Hewett et al. (2006). The agreement between the UKIDSS and ISAAC $J H K$ fluxes is very good.

\section{RESULTS AND MODELING}

The results of the Spitzer, UKIDSS, and ISAAC photometry are given in Table 2 along with their uncertainties. Figure 2 shows the Spitzer and ISAAC photometry, and the ISAAC spectroscopy plotted on the same axes as the UV and optical data from Gänsicke et al. (2006). The model of the expected flux from the white dwarf (taken from the same paper) is also shown. There is an substantial flux excess over the expected emission from the white dwarf, starting in the $K$ band and increasing out to $16 \mu \mathrm{m}$, before dropping at $24 \mu \mathrm{m}$.

We attempted to model this excess with a simple optically thick, geometrically thin dust disk model with a temperature profile $T_{\text {disk }} \propto r^{-\beta}$, with $\beta=3 / 4$, as previously used, e.g., by Jura (2003), Becklin et al. (2005), and Brinkworth 
Table 3

Parameters for the Dust Disk Model Discussed in Section 3

\begin{tabular}{lr}
\hline \hline Parameter & Value \\
\hline Inner disk temperature $(\mathrm{K})$ & 1670 \\
Inner disk radius $\left(R_{\mathrm{WD}}\right)$ & 18 \\
Outer disk temperature $(\mathrm{K})$ & 450 \\
Outer disk radius $\left(R_{\mathrm{WD}}\right)$ & 107 \\
Disk inclination $(\mathrm{deg})$ & 70
\end{tabular}

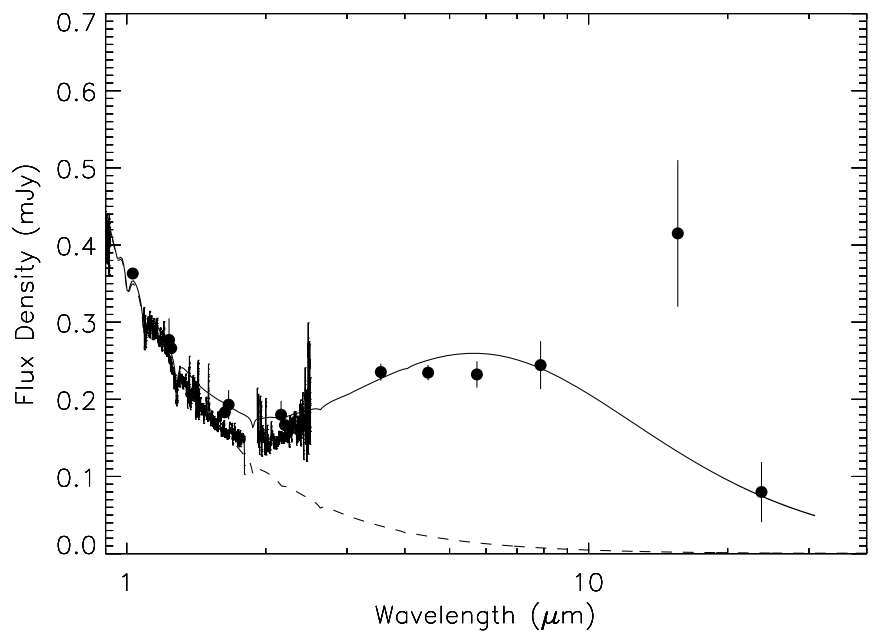

Figure 3. IR SED of SDSS $1228+1040$, compared with a model consisting of a white dwarf (dashed line) and an optically thick, geometrically thin circumstellar dust disk (dash-triple-dotted line). The significant excess at $16 \mu \mathrm{m}$ may be due to a strong silicate emission feature. For more details see Section 3.

et al. (2007). In principle, $\beta$ can take values different from $3 / 4$ depending on the size of the dust grains, as the grains will cool inefficiently once that the grain size is comparable or smaller than the peak wavelength of the Planck function at the grain temperature, $r_{\text {grain }} \lesssim b / T$ (with $b$ the Wien constant, for details; see Brinkworth et al. 2007). In practice, however, we found that values significantly different from $3 / 4$ do not produce viable results. As in Brinkworth et al. (2007), we therefore adopt this value for our modeling. The input parameters are given in Table 3.

The model is shown in Figure 3. While it fits the near-IR, IRAC, and MIPS points well, the $16 \mu \mathrm{m}$ flux density is $2.0 \sigma$ greater than predicted. This $16 \mu \mathrm{m}$ excess may be explained by the presence of a significant silicate emission feature at $10 \mu \mathrm{m}$, as seen in the similar dusty white dwarf GD 362. Jura et al. (2007b) found that this emission feature has a strong red wing that, if present in SDSS 1228+1040, would contribute significantly to the IRS Peak-Up band (which has a passband from 13 to $18.5 \mu \mathrm{m}$ ). It is possible that such a feature would also affect the $8 \mu \mathrm{m}$ flux density, but the blue wing of the silicate feature in GD 362 was less pronounced than the red wing, so the effect is expected to be minor (of order a few \%). This is not currently accounted for in the model, but could explain the slight rise seen at $8 \mu \mathrm{m}$ from the rest of the IRAC bands.

\section{DISCUSSION AND CONCLUSIONS}

The ISAAC, UKIDSS, and Spitzer Space Telescope data show a noticeable IR excess in SDSS $1228+1040$, in excess of the flux density expected from the white dwarf alone. The observed SED provides clear and compelling evidence for the presence of a substantial dusty component to the debris disk, in addition to the gaseous component discovered by Gänsicke et al. (2006), indicating that both gaseous and dusty debris material can coexist around a white dwarf. The presence of dust around SDSS 1228+1040 implies that debris disks can survive around relatively hot parent white dwarfs, contrary to the earlier suggestion by Kilic et al. (2006) and von Hippel et al. (2007), based on a smaller sample of white dwarfs with debris disks, that white dwarfs hotter than around $15,000 \mathrm{~K}$ should sublimate any orbiting dust disk.

Our model shows that an optically thick dust disk model can reproduce the SED seen in SDSS 1228+1040, except for the $16 \mu \mathrm{m}$ point, which we currently attribute to the red wing of a strong silicate feature at $10 \mu \mathrm{m}$, as seen in similar systems (e.g., GD 362). We calculated a lower-limit estimate of the mass of dust in the optically thick disk using the generic mass absorption coefficient used by Beckwith et al. (1990) of

$$
\kappa_{\nu}=0.1\left(v / 10^{12} \mathrm{~Hz}\right)^{\beta} \mathrm{cm}^{2} \mathrm{~g}^{-1},
$$

where we follow their example and adopt $\beta=1$ (see Brinkworth et al. 2007, for a more in-depth discussion). This leads to a disk mass of at least $1 \times 10^{22} \mathrm{~g}$, equivalent to $\sim 1.3 \times 10^{-4} M_{\text {Moon }}$ or $\sim 0.01$ times the mass of Ceres.

There are, however, two main issues with our disk model. Firstly, in order to match the rise from the $K$ band to the IRAC channel 1 point at $3.6 \mu \mathrm{m}$, we find that the inner temperature of the dust disk has to be around $1670 \mathrm{~K}$, which is hotter than the expected sublimation temperature of the dust grains (around 1200-1400 K). Moreover, if we estimate the inner radius of the gaseous disk from Gänsicke et al. (2006) using $R_{\text {innerdisk }} \sim\left(V_{\text {peak }} / V_{\text {max }}\right)^{2} \times R_{\text {outerdisk }}$, where $V_{\text {peak }}$ is the velocity of the line peaks and $V_{\max }$ is the maximum velocity at which emission is seen, then we find that the inner radius of the dusty disk at this temperature is smaller ( $\left.18 R_{\mathrm{WD}}\right)$ than the inner radius of the gaseous disk ( $\left.\sim 27 R_{\mathrm{WD}}\right)$. It is possible that the inclusion of a 2- or 3-zone, or warped disk model would allow us to fit the data using a cooler inner disk temperature (and therefore larger inner dust disk radius) but the lack of data points in the mid-IR would make it impossible to usefully constrain such a model, and so we do not pursue it further.

Second, we find that the implied outer radius of the dusty disk $\left(\sim 107 R_{\mathrm{WD}}\right)$ coincides almost perfectly with the outer radius of the optically thin gaseous disk $\left(\sim 108 R_{\mathrm{WD}}\right)$, as derived by Gänsicke et al. (2006), so the gaseous dust disk permeates throughout the dusty component. This is not unexpected in itself, as the sublimating dust will likely replenish the gas disk. It is possible, however, that the lifetime of the dust grains will be relatively short due to the increased viscosity. Gänsicke et al. (2007) determined the photospheric abundance of $\mathrm{Mg}$ in SDSS1228+1040 to be $\log (\mathrm{Mg} / \mathrm{H})=-4.58 \pm 0.06$. Using the prescription of Koester \& Wilken (2006), and assuming that hydrogen fraction in the circumstellar material is depleted by a factor 100 with respect to its solar value, we estimate an accretion rate of $\dot{M} \simeq 3 \times 10^{9} \mathrm{~g} \mathrm{~s}^{-1}$. This is within the range of accretion rates obtained by Farihi et al. (2008) following a similar approach for the currently known sample of metal-polluted white dwarfs with dusty debris disks. We stress, however, that this estimate of the accretion rate should likely to be a strict upper limit, as $\dot{M}$ is inversely proportional to the hydrogen depletion in the circumstellar material, and the factor 100 assumed above is probably underestimated (Friedrich et al. 1999; Gänsicke et al. 2008). Combining the accretion rate with the estimate of the disk mass from above, we estimate a lower limit on the lifetime of the disk of $\sim 1000 \mathrm{yr}$. 
We thank the participants of the Winter AAS 2008 White Dwarf Splinter Session for their extremely helpful discussions and suggestions. This work is based on observations made with the Spitzer Space Telescope, which is operated by the Jet Propulsion Laboratory, Caltech, under NASA contracts 1407 and 960785 . This work makes use of data products from the Two Micron All Sky Survey, which is a joint project of the University of Massachusetts and IPAC/Caltech, funded by NASA and the NSF. We acknowledge and thank the referee Jay Farihi for his detailed report.

\section{REFERENCES}

Adelman-McCarthy, J. K., et al. 2006, ApJS, 162, 38

Becklin, E. E., Farihi, J., Jura, M., Song, I., Weinberger, A. J., \& Zuckerman, B. 2005, ApJ, 632, L119

Beckwith, S. V. W., Sargent, A. I., Chini, R. S., \& Güsten, R. 1990, AJ, 99, 924

Brinkworth, C. S., et al. 2007, ApJ, 659, 1541

Debes, J. H., \& Sigurdsson, S. 2002, ApJ, 572, 556

Farihi, J., Jura, M., \& Zuckerman, B. 2009, ApJ, 694, 805

Farihi, J., Zuckerman, B., \& Becklin, E. E. 2008, ApJ, 674, 431

Fazio, G., et al. 2004, ApJS, 154, 10

Friedrich, S., Koester, D., Heber, U., Jeffery, C. S., \& Reimers, D. 1999, A\&A, 350,865

Gänsicke, B. T., Koester, D., Marsh, T. R., Rebassa-Mansergas, A., \& Southworth, J. 2008, MNRAS, 391, L103
Gänsicke, B. T., Marsh, T. R., \& Southworth, J. 2007, MNRAS, 380, L35

Gänsicke, B. T., Marsh, T. R., Southworth, J., \& Rebassa-Mansergas, A. 2006, Science, 314, 1908

Graham, J. R., Matthews, K., Neugebauer, G., \& Soifer, B. T. 1990, ApJ, 357, 216

Hambly, N. C., et al. 2008, MNRAS, 384, 637

Hewett, R. J., Warren, S. J., Leggett, S. K., \& Hodgkin, S. T. 2006, MNRAS, 367,454

Houck, J., et al. 2004, ApJS, 154, 18

Jura, M. 2003, ApJ, 584, L91

Jura, M., Farihi, J., \& Zuckerman, B. 2007a, ApJ, 663, 1285

Jura, M., Farihi, J., \& Zuckerman, B. 2009, AJ, 137, 3191

Jura, M., Farihi, J., Zuckerman, B., \& Becklin, E. E. 2007b, AJ, 133, 1927

Kilic, M., von Hippel, T., Leggett, S. K., \& Winget, D. E. 2005, ApJ, 632, L115

Kilic, M., von Hippel, T., Leggett, S. K., \& Winget, D. E. 2006, ApJ, 646, 474

Koester, D., Provencal, J., \& Shipman, H. L. 1997, A\&A, 320, L57

Koester, D., \& Wilken, D. 2006, A\&A, 453, 1051

Lawrence, A., et al. 2007, MNRAS, 379, 1599

Makovoz, D., Roby, T., Khan, I., \& Booth, H. 2006, Proc. SPIE, 6274, 10

Reach, W. T., Kuchner, M. J., von Hippel, T., Burrows, A., Mullally, F., Kilic, M., \& Winget, D. E. 2005, ApJ, 635, L161

Rieke, G., et al. 2004, ApJS, 154, 25

Rousselot, P., Lidman, C., Cuby, J.-G., Moreels, G., \& Monnet, G. 2000, A\&A, 354, 1134

Telesco, C. M., Joy, M., \& Sisk, C. 1990, ApJ, 358, L17

Tokunaga, A. T., Becklin, E. E., \& Zuckerman, B. 1990, ApJ, 358, L21

von Hippel, T., Kuchner, M. J., Kilic, M., Mullally, F., \& Reach, W. T. 2007, ApJ, 662,544

Zuckerman, B., \& Becklin, E. E. 1987, Nature, 330, 138 